\title{
EL ENCABALGAMIENTO ( $L E$ ENJAMBEMENT): UNA APROXIMACIÓN A SU SIGNIFICADO E IMPORTANCIA EN EL TEXTO HOMÉRICO DE LA ODISEA, CANTOS 1, 5 Y 13
}

\author{
José García López \\ Universidad de Murcia \\ joseglopez@movistar.es
}

\section{RESUMEN}

El autor realiza su estudio en el marco del acercamiento a la poesía de la épica homérica y la oral poetry. El trabajo selecciona y comenta los tres cantos primeros de La llamada

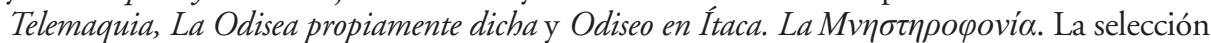
se realiza con la búsqueda de aquellos lugares, en donde el autor épico emplea el llamado "the Enjambement (The Runover) integral necessary". Se busca analizar y, en su caso, defender y valorar el empleo de este recurso de estilo por un único autor de la Odisea. Por razones de espacio, sólo se tendrán en cuenta aquellos ejemplos en los que la figura del encabalgamiento se realiza por medio de una forma verbal.

Palabras Clave: Odisea 1, 5 y 13, estilo épico, El Encabalgamiento.

THE ENJAMBEMENT: AN APPROACH TO ITS MEANING AND IMPORTANCE

IN THE HOMERIC TEXT OF THE ODYSSEY, BOOKS 1, 5 AND 13

\section{ABSTRACT}

The author develops his study in the framework of the approach to the poetry of the Homeric epic and the oral poetry. The work selects and comments on the first three songs of The call

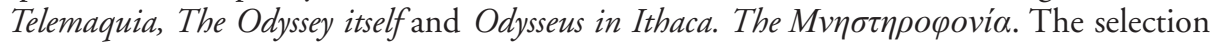
is made with the search of those places where the epic author uses the so-called The Enjambement (The Runover) integral necessary. The aim is to analyze and, where appropriate, defend and value the use of this style as a resource by a single author of the Odyssey. For space reasons only those examples will be taken into account in which the figure of The Enjambement is carried out by means of a verbal form.

KeYwords: Odyssey 1, 5 and 13, Epic Style, The Enjambement.

\section{A MODO DE PRÓLOGO}

Antes de iniciar nuestra aproximación a una figura, El Encabalgamiento, sobre los datos que nos ofrecen en los textos los cantos 1, 5 y 13 de la Odisea, queremos 
hacer algunas precisiones, que, de alguna manera, sitúen al posible lector en los pasajes que nos proponemos analizar sobre esta figura de estilo, que hemos elegido, entre otras muchas posibles, del texto épico.

En primer lugar, al tratarse de un texto poético, el estudio de su forma literaria ha de ser necesaria y naturalmente distinta a la que deberíamos tener en cuenta, si la acción la lleváramos a cabo sobre un texto en prosa ${ }^{1}$. Partimos afortunadamente de un acercamiento muy personal a través de los datos que nos ha facilitado una traducción propia anotada, en curso de publicación, del texto de la Odisea. Por ello hemos podido tener en cuenta la obra completa en traducción castellana personal del gran poema épico y sobre ella seleccionar los versos en estudio a partir de la tradicional clasificación del poema: I. La llamada Telemaquia (con el viaje iniciático de Telémaco, cantos 1-4). II. La Odisea propiamente dicha (con los relatos personales

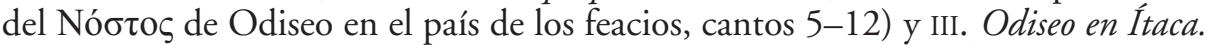
La $\mu v \eta \sigma \tau \eta \rho \varphi o v i \alpha$, la matanza de los pretendientes (es decir, Odiseo en Ítaca y su vengan$\mathrm{za}$, matando a sus siervos y esclavas infieles y a los pretendientes de Penélope, cantos 13-24)2. Por lo demás, para llevar a cabo esta recogida de material, lo haremos, de acuerdo a nuestro convencimiento, eligiendo la definición del término de la que parte el profesor de Cambridge, Kirk, al tratar en Homero el problema del encabalgamiento, y que define así: "The carrying over of the sentence of one verse into the next, envolving an overrunning of the verse-end $\aleph^{3}$. Por todo ello, a partir de aquí,

${ }^{1}$ Sin dejar de valorar las consideraciones, por ejemplo, de Dionisio de Halicarnaso, Comp. 26, 11-15, sobre la posible semejanza entre prosa y poesía, si ésta última así lo posibilita, por ejemplo, en la disposición y en el orden de las palabras y que no haga coincidir el final de los $\kappa \tilde{\omega} \lambda \alpha$ con el final del verso, $\sigma \tau i ́ \chi \chi \zeta$, , como podemos añadir ahora, se exige en la figura del encabalgamiento, que, según estas afirmaciones de Dionisio, acercaría estas dos formas de componer en griego y que ahora nos proponemos estudiar nosotros.

${ }^{2}$ Cf., por ejemplo, Seek, G. A. (2004): Homer. Eine Einführung, Stuttgart, p. 198: Eine aüssere Dreiteiligkeit der Odyssee:

Buch 1-4 Telemachie

5-12 Odysseus unterwegs (Odysseus bei den Phäaken, p. 229)

13-24 Odysseus in Ithaka (Die Freiergefahr, p. 252)

${ }^{3}$ En Kirk, G. S. (1976): Homer and Oral Poetry, Cambridge University Press, Cambridge, p. 147. Por su parte Parry, M: «The distinctive Character of Enjambement in Homeric Verse», TAPhA 60 (1929) pp. 200-220, consideraba el encabalgamiento como "a self-explanatory term", por lo que no ofrece una definición formal, aunque podemos consultar con gran utilidad para nuestro propósito lo que escribe en la p. 203, como resultado del análisis que hace en las páginas 201-203, al comentar un pasaje sobre la Odisea, 14, 1-7, junto al Fr. 696, 1-7 Nauck del Télefo de Eurípides y al Fr. 38, 1-23 Page, sobre Dánae de Simónides, del escritor griego Dionisio de Halicarnaso, Comp. 26, 11-13, que estudia la relación y posible semejanza entre prosa y poesía épica. Con ello analiza las tres formas en las que se puede presentar una oración en el verso homérico, llegando a la conclusión de que de las tres formas posibles sólo en dos de ellas se puede hablar de enjambement: Una, cuando el sentido, no la frase u oración, aparece completado o explicado en el verso siguiente con un epíteto o una frase adverbial

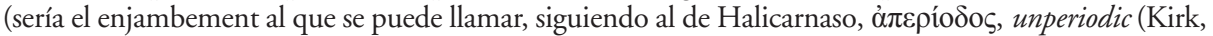
1976: 147, propondrá, al creer confuso este término, el adjetivo "progressive"), y, dos, cuando el verso 
hemos escogido para nuestro análisis sólo los versos, que nos facilitan los tres cantos elegidos, donde tomaremos cada uno como ejemplo representativo por su posición inicial en las tres partes en las que se acepta dividido el poema. De este modo, pensamos, dispondremos de los ejemplos apropiados a la hora de decidir nuestra postura y sobre ella sacar posibles conclusiones.

Como acabamos de indicar, hemos hecho una doble elección, cuya dificultad reconocemos, como, por otra parte, lo es todo estudio que pretenda analizar cualquiera de los múltiples aspectos posibles en un acercamiento al texto épico atribuido a Homero y por lo que el nuestro no podía ser una excepción. Una de las elecciones ya la hemos indicado, así como hemos expuesto brevemente las razones para su selección, usando, sin pretenderlo, la figura tan homérica del v̌ La otra, y desde luego la primera y la más importante, ha sido nuestra elección de la forma literaria del encabalgamiento, le enjambement, que, como acabamos de decir, siempre estuvo en primer lugar, desde el momento en que decidimos realizar un estudio de una de las figuras de estilo ${ }^{4}$ más recurrentes y también muy analizadas 5

termina al final de un grupo de palabras, en el que, sin embargo no finaliza el pensamiento, o termina en medio de un grupo de palabras, en ambos casos tendríamos el "enjambment integral necessary". En efecto, en las pp. 146-155 el profesor Kirk escribe enjambment no enjambement. No obstante, en la nota 8 de este artículo M. Parry nos dice que él entenderá el enjambement en su sentido más amplio como «that of running over of the sentences from one line to another». Sobre la diferencia entre formas de composición en verso y en prosa, cf. también Demetrio, Eloc. I 1.

${ }^{4}$ Y que personalmente hemos intentado señalar en las notas a nuestra traducción de la Odisea y que se suman a la extraordinaria valoración como gran poeta del autor de este poema épico, y que, entre otras, destacaríamos las siguientes clases: Anticipación ( $\pi \rho \lambda_{\lambda} \eta \psi 1 \varsigma, O d .12,40$ ss.), Retrospección

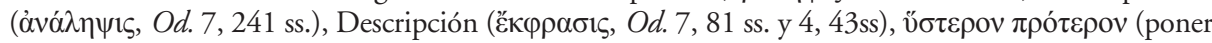

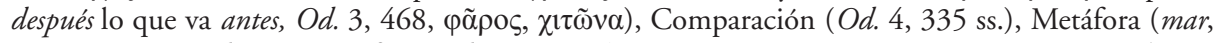

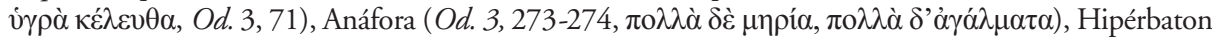

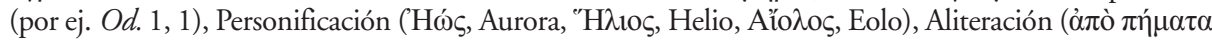

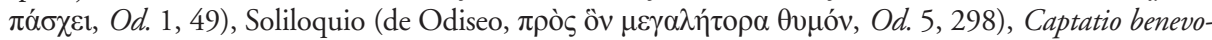
lentiae, (Od. 5, 445-450, Ringkomposition (1, 428-434), etc. y, naturalmente, la figura del Encabalgamiento, que, con frecuencia comparte versos con algunas de estas figuras, ya nos sale al paso en los dos primeros versos de la Odisea y que hemos elegido para nuestro estudio. Sobre el lenguaje poético en Homero, entre otros, cf. Meister, Karl (1921): Die homerische Kunstsprache, Leipzig, B. G. Teubner, pp. VIII+262, sobre todo p. 28, nota 1, donde resalta la frecuencia del Enjambement en Virgilio frente a la búsqueda de final de contenido al final de verso en Homero, y Bassett, S. E. (1938, 2003 $\left.{ }^{12}\right)$ : The Poetry of Homer, Berkeley, University Press, pp. 273, sobre todo los capítulos IV y VI.

${ }_{5}^{5}$ Sólo algunos ejemplos, prácticamente todos de origen anglosajón, citando únicamente uno de los también numerosos trabajos, siguiendo los trabajos de Parry y su alumno Lord, sobre Épica eslava y Homero: Bassett, S. E.: «The So-Called Emphatic Position of The Runover Word in the Homeric Hexameter», TAPhA 57 (1926), pp. 116-148. Parry, M.: «The Distinctive Character of Enjambement in Homeric Verse», TAPhA 60 (1929), pp. 200-220. Lord, A. B.: «Homer and Huso III: Enjambement in Greek and southslavic Heroic Song», TAPhA 79 (1948), pp. 113-124. Kirk, G. S. (1976): Homer and the Oral Poetry, University Press, Cambridge 1976, pp. 146-174. Clayman, Dee Lesser and Van Norwick, Thomas: «Enjambement in Greek Hexameter Poetry», TAPhA, 107 (1977), pp. 85-92, que critican la obra de M. Parry por querer trasladar sus resultados a toda la poesía épica griega. Barnes, Harry. R.: 
en la composición oral del texto homérico, conscientes siempre de su importancia y las múltiples relaciones estudiadas con el llamado estilo homérico y que a veces tendremos que mencionar, pero que naturalmente no abordaremos.

En relación con lo anteriormente expuesto tenemos personalmente presente, al iniciar nuestro estudio, la acertada y para nuestro análisis también valiosa precisión del estudioso alemán Gustav Adolf Seeck, von 1981 bis 1999 Professor für Klassische Philologie (Gräzistik) an der Universität Frankfurt a. M., cuando escribe, en su obra citada en la nota 2 , p. 58 , que:

«Es war eine verständliche, aber sehr einseitige Idee der Forschung, bei Homer überall das "Typische" zu betonen und darüber zu vergessen, dass jede Verwundung, jedes Gleichnis, jede "typische Szene" anders ist. Diese geradezu unerschöpfliche Variationskunst ist zweifellos eine Folge des Übergangs zur schriftlichen Grossform».

Es decir, es comprensible, aunque también unilateral en la investigación del texto homérico, la idea de poner de relieve lo "típico", olvidando que cada herida, cada comparación ${ }^{6}$ o cada escena típica es diferente y que esta variación artística es,

«Enjambement and Oral Composition», TAPhA 109 (1979), pp. 1-10. Clark, Matthew: «Enjambment and Binding in Homeric Hexameter», Phoenix 48.2 (Sommer, 1984), pp. 95-114. Bakker, Egbert J.: «Homeric Discourse and Enjambement: A Cognitive Approach", TAPhA 120 (1990), pp. 1-21. Parry, A. (ed.), (1987): The Making of Homeric Verse, Oxford, donde, entre otros trabajos de su padre Milman, se recoge el artículo sobre el Enjambement, antes citado, en pp. 251-265. C. Higbie, C. (1990): Messure and Music: Enjambement and Sentence Structure in the Iliad, Oxford, a la que preguntaríamos dónde cita en su bibliografía, pues no hemos sido capaces de encontrar, alguna obra o tratado de música griega, y en qué página de su libro se plantea la relación entre metro, ritmo y música en su acercamiento al hexámetro de Homero. Dukat, Zdeslav: «Enjambement as a Criterion for Orality in Homeric and South Slavic epic Poetry», Oral Tradition, 6/2-3(1991), pp. 303-315 (publicada como Journal at Harvard University, desde 1986). Personalmente hemos de aceptar que sólo conocemos una obra anterior en lengua alemana y citada en nota anterior, nos referimos a K. Meister, 1921, p 28, nota 1, donde habla y compara el uso de Das Enjambement en Homero y Virgilio.

${ }^{6}$ Recordemos, por ejemplo, la variedad, conveniencia y, a la vez, diferencia entre las cinco breves comparaciones o símiles tomados, como siempre, del entorno vital, que tan bien conoce el poeta, y con los que Homero, en un verdadero "nido", adorna y prepara su introducción al prolijo y detallado catálogo de las naves en la Ilíada, II vv. 455-483. Estos 32 versos, que refieren el brío y el deseo de los aqueos para ir al combate contra los troyanos, van precedidos de un breve relato sobre cómo la hueste de los aqueos es animada a la lucha por la ojizarca Atenea, para, en seguida, comparar: I, el brillo del bronce de los aqueos con un voraz fuego que abrasa un bosque, 455-458; II, las tribus de los combatientes griegos con las numerosas razas de las volátiles aves, ganso, grullas o cisnes, que revolotean por las cauces del Caístro, 459-468; III, los aqueos de larga cabellera con las espesas moscas que vagan errantes por el pastoril establo, cuando las tinajas rezuman de leche, 469-473; IV, los jefes griegos que ordenan su tropa con los cabreros que agrupan y ordenan sus rebaños de cabras, 474-479, y finalmente, $v$, al Atrida Agamenón, que reúne a los aqueos, con el buey y el toro que sobresalen entre las vacas reunidas a su alrededor. A continuación tenemos la plegaria del aedo a las Musas para que le enumere los caudillos, tropas y naves que los transportaron hasta la llanura troyana y el famoso catálogo de naves y caudillos griegos, que las comandaban, de los que, en el mismo canto 2, 798 801, la diosa mensajera Iris pondera su multitud ante Príamo y Héctor, diciendo que son: "como hojas o granos de arena en gran cantidad". 
sin duda alguna, consecuencia de la transición (de la composición original oral) a la forma (Grossform, "letras mayúsculas") escrita".

Así, al analizar la figura del Enjambement, observaremos, en efecto, su frecuente aparición, pero también su propia razón de ser en una obra poética compuesta y transmitida de forma oral (oral poetry) ${ }^{7}$, que por naturaleza tiende a ser desigual y también a evitar las frases largas y complejas ${ }^{8}$, y su peculiaridad en los distintos lugares en los que aparece, ¿¿un verdadero Leimotiv?, y que nos proponemos someter a estudio.

Inicialmente los ejemplos reunidos ${ }^{9}$ en nuestra lectura de los tres cantos elegidos, entendiendo que la figura en estudio se corresponde con el encabalgamiento del final de un verso hacia el siguiente (sin signo de puntuación alguno) sobre el que el poeta vuelca y termina su actual pensamiento y secuencia gramatical, nos ha dado la siguiente clasificación: 1. FORMAS VERBALES; 2. NOMBRES COMUNES Y NOMBRES PROPIOS; 3. ADJETIVOS; 4. ADVERBIOS, y, por último, 5. GIROS PREPOSICIONALES, de las que sólo podremos analizar individualmente por razones obvias de espacio, las que se refieren al uso de FORMAS VERBALES. Señalaremos, también, que, atendiendo a nuestra postura ante la figura elegida, sólo analizaremos aquellos ejemplos que se atengan a la definición del llamado necessary enjambement, por Milman Parry en su obra y página citadas en nuestra nota número tres, es decir, "cuando el verso termina al final de un grupo de palabras, en el que, sin embargo no finaliza el pensamiento, o termina en medio de un grupo de palabras», aunque, más exactamente tendremos en cuenta el tipo que Kirk ${ }^{10}$ llama "integral necessary", que se distingue

${ }^{7}$ Cf., por ej., la publicación más completa, pensamos hasta ahora, sobre la composición oral de la épica homérica, de Kirk, op. cit., en la que dedica unas páginas, 146-164, al tema que ahora nos ocupa, y con las que, escribe, se propone volver a examinar los diferentes tipos de enjambement en el hexámetro de Homero (Iliada y la Odisea), así como haciendo incursiones a la épica romana (Verg. Aen. 4, 365-387) y a la inglesa (Milton, Paradise Lost, II 539-546). Con estos datos decide, centrándose en el canto 16 de Ilíada. y a partir de los trabajos de Parry y Lord, pp. 148-155, llamar "periodic" al ejemplo del verso que termina con una cláusula subordinada, con coma, y es completada por la frase principal en el verso siguiente, $I l$. 16, 36, e "integral necessary", sin coma, para el caso en que la frase empieza en un verso y termina al principio del verso siguiente, por ej. en el mismo canto 16, v. 81s. y 16, 46s. Además, cuando dos palabras, que van unidas por naturaleza (preposición o epíteto, con el nombre que rige o al que califica), colocados en versos distintos, llama "violent", ej: $I l$. 16, 62s., 104s. y 396s, y precisando que la diferencia entre el caso 3, necessary, y el caso 4, violent, puede ser subjetivo. (pg. 150). Finalmente, sugiere el término Progressive para designar al encabalgamiento llamado unperiodic, por M. Parry, a partir del análisis de Od. 14, 1-7, que realiza D.H., Comp. 26, 12.

${ }^{8}$ Kirk, G. S., Op. cit. p. 205.

${ }^{9}$ Finalmente, por razones de espacio, sólo hemos sometido a nuestro análisis los ejemplos de los tres libros citados en los que la figura el encabalgamiento "integral necessary" se realiza a través de empezar el segundo verso del hemistiquio estudiado con una forma verbal. Los demás casos, nombres comunes y propios, adjetivos, adverbios y frases preposicionales, también los tengo localizados, y, en ellos puedo también apoyar mi análisis, si fuera necesario.

${ }^{10}$ Op. cit. p. 148. 
por carecer de coma ${ }^{11}$ en el primero de los versos estudiados del canto 16 de la Iliada ${ }^{12}$.

\section{ANÁLISIS}

\subsection{LA LLAMADA TELEMAQUIA (OD. 1-4)}

Comencemos, pues, por el primer ejemplo, que nos ha salido al encuentro en nuestro acercamiento al texto del primer canto de la Odisea, en la llamada

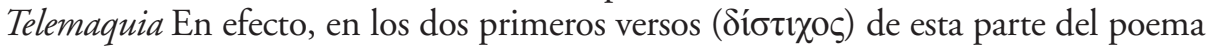
épico tenemos el siguiente texto:

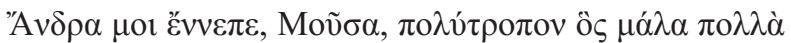

$\pi \lambda \alpha ́ \gamma \chi \theta \eta$,

«Háblame, Musa, del hombre de multiforme ingenio que muy mucho anduvo errante».

El texto griego, del llamado Proemio de este canto épico, en su primer verso, que finaliza sin coma, nos ofrece, desde un primer momento una construcción de ritmo

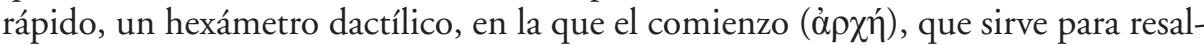
tar lo que se propone obtener de la divinidad, lo ocupan el sustantivo "Av $\delta \rho \alpha$ y el pronombre $\mu \mathrm{ol}$, en clara inversión ( su parte central ( $\mu \varepsilon \dot{\sigma o v}$ ), que suele destacar la palabra que se coloca en ella, la ocupa

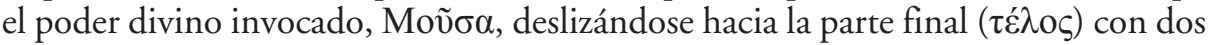
formas adverbiales, que se desbordan hacia el verso segundo, con ritmo dactílico parecido, hasta la forma verbal $\pi \lambda \alpha ́ \gamma \chi \theta \eta \eta$. Este verbo, en claro encabalgamiento "integral necessary", completa la frase relativa que comenzada en el verso anterior con un ôs, su sujeto, y con el que el aedo completa y explica el adjetivo-epíteto, $\pi$ o $\lambda v ́ \tau \rho o \pi o v$, de 'A $v \delta \rho \alpha$, objeto principal de la súplica a la Musa. Vemos, por tanto, que la figura objeto del presente estudio se enmarca en una sucesión de rasgos, figuras poéticas, que la acogen con naturalidad y que la forma de la oral poetry, en la que sin duda el aedo que compuso este proemio, lo acepta con sencillez, para llegar al final

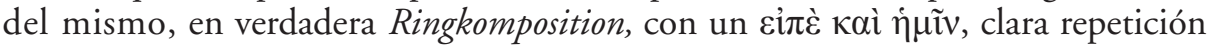

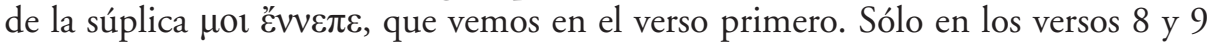

${ }^{11}$ Pero tengamos en cuenta que, según el editor del texto homérico, con frecuencia las comas y en general los signos de puntuación no coinciden o faltan en un ejemplo y sí lo están en otro. Cf., por ej., las conocidas ediciones de la Ilíada de Mazon y Monro-Allen.

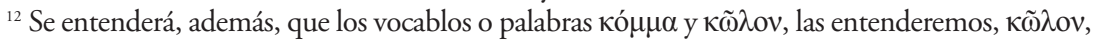
en su sentido general como miembro de un período y cláusula al final de un verso ( $\sigma \tau i ́ \chi \varnothing \varsigma)$, mientras que una אó $\mu \mu \alpha$ será igual a nuestra "coma", es decir, expresión más pequeña que un $\kappa \tilde{\omega} \lambda o v$ y que también entenderemos como "frase". 
de este mismo proemio ${ }^{13}$, y antes de pasar al canto 5 , señalaremos un nuevo caso de encabalgamiento integral necessary, cuando el aedo recita los mencionados versos 8 y 9:

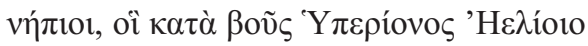

हैбOtov.

«infelices, que las vacas de Helios Hiperión

devoraron»:

y que en el original griego, con ritmo dactílico total, como en el caso del verso primero y sin coma igualmente al final del verso 8 , se ve reforzada la figura en estudio

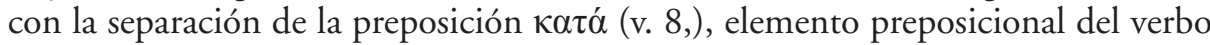

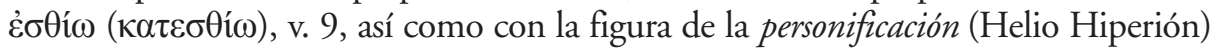
de la fuerza natural, que representa el sol.

En este primer canto encontramos que al menos en seis de los versos analizados, naturalmente al principio del segundo de los versos señalados, el encabalgamiento "integral necessary" se relaciona y señala con una forma verbal al contenido principal de esta primera parte, en La llamada Telemaquia, hemos encontrado 13 ejemplos en los que se da la misma proporción de 7 frente a 6, por tanto, muy parecida

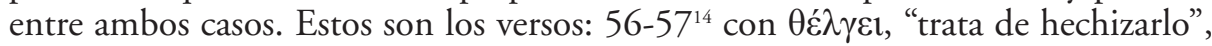
en las palabras con las que comunica y reprocha la diosa Palas Atenea a su padre Zeus y los demás dioses reunidos, excepto Posidón, en su palacio, su actitud ante las desgracias de su protegido Odiseo, y que veremos repetidas de nuevo en el canto quinto,

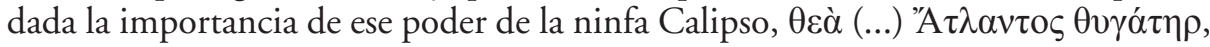
que retiene hechizado a Odiseo, ya en su séptimo año de estancia en su isla; $341-342^{15}$ con $\tau \varepsilon i ́ p \varepsilon 1$, "atormenta", en boca de Penélope que se queja al aedo Femio por cantar el triste regreso de los griegos, tras la destrucción de Troya, un dolor de la reina por su esposo tanto tiempo ausente que domina y está presente en toda esta primera parte

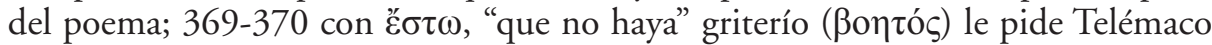
a los pretendientes de su madre, pues no le agrada lo que puedan comentar los súbditos de su padre ausente, que no comprenderían la alegría que escuchaban; 376-377 16 con $\check{\varepsilon} \mu \mu \varepsilon v \alpha$ l, "es", precedido en el verso anterior por la oración principal condicional

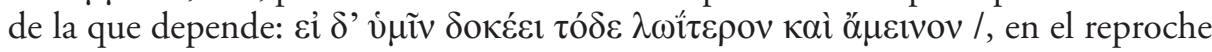

${ }^{13}$ En efecto, sólo en seis más de los casos estudiados, con una forma verbal, podremos señalar cierta importancia e hincapié en el contenido de esta primera parte, y no en los siguientes, donde igualmente hemos visto empleada la figura del encabalgamiento "integral necessary" y que son: 189-190; $191-192 ; 215-216 ; 262-263 ; 262-263$ у 410-411.

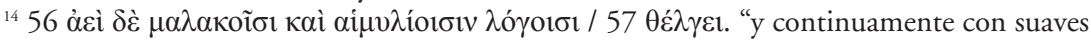
y aduladoras palabras trata de hechizarlo".

${ }^{15} 341 \lambda v \gamma \rho \tilde{\eta} \varsigma$, en el pecho mi corazón atormenta". "Triste" es el canto del aedo Femio.

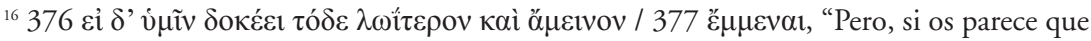
más provechoso y mejor / esto es". 
que Telémaco hace a los pretendientes que están consumiendo gratis los bienes de su ausente padre, y que inciden en la atmósfera opresiva y dolorosa que sufren Penélope y su hijo Telémaco, y que se nos describe en esta primera parte, como anun-

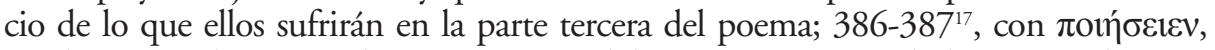
"ojalá (no) te haga”, en la contestación del más importante de los pretendientes, Antínoo, a Telémaco, que, en parte, hemos visto en versos anteriores, 376-380, y que nos dan a conocer la soberbia y la arrogancia de los pretendientes a la mano de Penélope, que piden al Cronión no haga al hijo de Odiseo su sucesor y rey de Ítaca; $421-422^{18}$ con $\tau \rho \varepsilon \psi \alpha ́ \alpha \varepsilon \varepsilon v o 1 ~ \tau \varepsilon ́ \rho \pi o v \tau o$, los pretendientes, al baile y al placentero canto "volviéndose, disfrutaban". Sin duda, el único quehacer de los engreídos pretendientes a lo largo de la Telemaquia, y que el poeta una y otra vez resalta con su soberbia

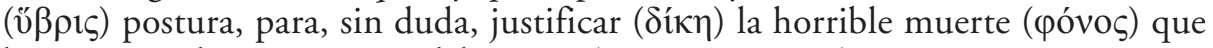
les espera en la tercera parte del poema ( $\mu \nu \eta \sigma \tau \eta \rho о \varphi o v i ́ \alpha)$.

\subsection{LA LLAMADA ODISEA PROPIAMENTE DICHA (OD. 5-12)}

Los dos primeros versos de una especie prólogo, con el que el aedo va a conectar con el final del canto anterior, que ha dejado a Telémaco en su lecho, meditando cómo haría mejor su proyectado viaje a Esparta y Pilo en busca de noticias sobre su padre, dicen así:

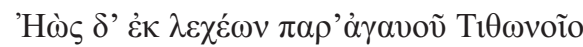

ö $\rho v 0 \theta$ ',

«Y Aurora del lecho del ilustre Titono

se levantaba,»

En estos dos versos ( $\delta$ í $\tau \imath \chi 0 \varsigma$ ), tenemos, en el primero, la figura de la personificación del fenómeno natural de la aurora, de las primeras luces del día, a la que incluso se le da un marido, Titono, de cuyo lecho se levanta, al parecer de mala gana, expresado con un cuarto y, sobre todo, con un quinto pie espondeos, como si fuera una mortal, por lo que hemos traducido, al castellano, como en el original griego, sin artículo. El primer verso, que termina sin coma, y que se desborda, "el Runover" de E. S. Bassett (1926), hacia el segundo verso nos sirve como otro ejemplo de la figura en estudio y que, siguiendo a Kirk, hemos llamado encabalgamiento "integral necessary". Además, en los primeros seis versos, como en el proemio del canto primero, se nos ofrece otro ejemplo, ahora en la forma de un participio, de esta misma figura en los versos 5-6, en los que, haciendo uso de una diríamos falsa retrospección

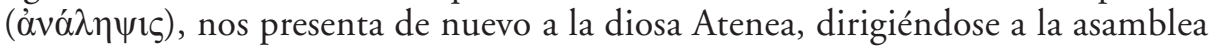

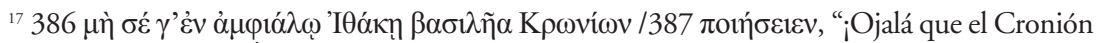
no te haga, por cierto, rey de Ítaca rodeada por el mar".

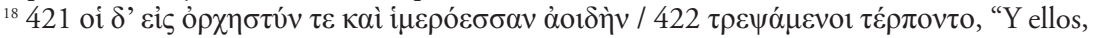
al baile y al placentero canto volviéndose, / disfrutaban". 
de los dioses para recordarles, como antes, su comportamiento injusto con su protegido, el héroe Odiseo y sus múltiples padecimientos, retenido ya tanto tiempo, siete años, por la ninfa Calipso, y que dicen así:

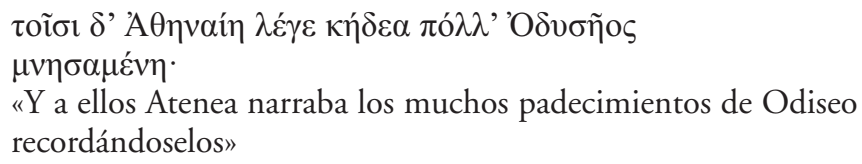

Los dioses escuchan, de nuevo, las quejas de Atenea, la poderosa hija de Zeus, que vuelve a repetir, expresándose con mayoría de vocales, 18 frente a 15 conso-

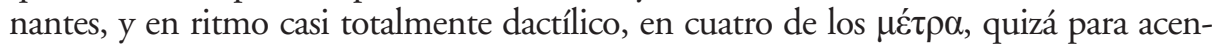
tuar e insistir en su disgusto por la injusticia que se está cometiendo con Odiseo, retenido demasiado tiempo y a la fuerza por la ninfa Calipso. La narración no nos lleva, como en el canto primero, con Atenea en la figura de un mortal, Mentes, a Ítaca y la posterior narración del viaje de Telémaco, convencido y ayudado por su diosa protectora. Ahora, en cambio, nos vamos con el mensajero de los dioses, Hermes, al hogar de la ninfa Calipso, para comunicarle la orden de Zeus, para que deje, como así lo hará, al héroe Odiseo, que, en efecto, emprenderá su largo y peligroso viaje

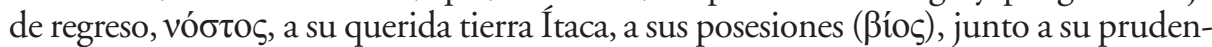

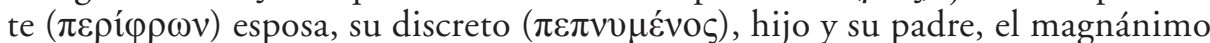
( $\mu \varepsilon \gamma \alpha \lambda \eta \dot{\tau} \omega \rho)$ Laertes. Vemos, así, que, sin aludir al viaje ya realizado por Telémaco a Esparta y Pilo, el aedo se dispone a narrar las aventuras, la verdadera Odisea, de su protagonista. La pregunta, entonces, sería: ¿necesita el posible oyente, o lector futuro, conocer ahora lo narrado en los cuatro primeros cantos o si sigue escuchando o leyendo el poema, al llegar al canto 15, con la partida de Telémaco de Pilo y el palacio de Néstor y más tarde, ya en el canto 16, la narración del encuentro entre el padre y el hijo en el aprisco de su siervo fiel Eumeo, se preguntará de dónde viene el padre y el hijo y en qué circunstancias han llegado a los dominios de su fiel e ilustre porquero

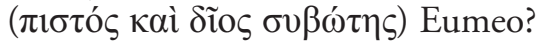

Como en el caso del canto primero observamos que el empleo en este quinto canto del encabalgamiento "integral necessary", en relación con el rico y variado contenido de esta segunda parte (cantos 5-13) lo hallamos, destacando algunos de sus aspectos más significativos, naturalmente en el segundo de los versos señalados, con una forma verbal, sólo en los siguientes versos ${ }^{19}: 14-15$, con $̌ ̋ \chi \varepsilon 1$, "retiene" referido

${ }^{19}$ No encontramos esta relación en los casos en los que también hemos hallado posibles ejemplos de la figura en estudio en este mismo canto quinto, también con una forma verbal, pero con un contenido no relacionado particularmente con las desgracias y el continuo vagar de Odiseo. Se trata de los versos siguientes: 47-48; 56-57; 60-61; 72-73; 149-150; 160-161; 252-253; 343-344; 363-364; 411-412; 430-431; 441-442; 456-457 y, finalmente, 461-462. En número, en efecto, mayor que en los casos anteriormente analizados, en la proporción de 13 a 8 , contando el ejemplo del verso primero, cuyo contenido hemos comentado y que nos lleva a la bella imagen de Aurora abandonando con verdadera desgana (quinto pie espondaico), el lecho del bello Titono. 
a la negativa de la ninfa Calipso de dejar partir a Odiseo; 57-68, con vaĩev, "habi-

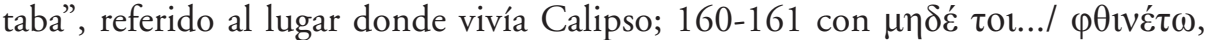
"no consumas", el ruego de Calipso a Odiseo, para que no pase sus días consumiéndose con la tristeza y añoranza por su querida patria y su prudente esposa; 270-271, con ท̆ $\mu \varepsilon v o \varsigma$, "sentado", con el timón gobernaba (Odiseo), con habilidad la balsa, recordándonos la pericia de Odiseo en las cosas relacionadas con el mundo

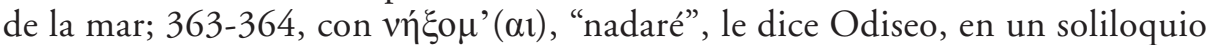
a su ánimo, mencionando una de los recursos empleados en su constante vagar

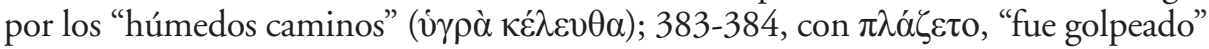
por una enorme ola, afrontando los naturales y constantes sufrimientos del marinero (de Odiseo), privado de su querida nave y sus desgraciados compañeros y, por último,

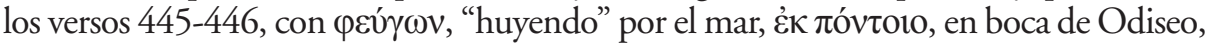
que huye de las iras de Posidón, y que se dirige con su plegaria al soberano que supone personifica el río, a cuya desembocadura ha llegado nadando, auxiliado una vez más por su diosa protectora Atenea, río que se hallaba en la última etapa de su continuo

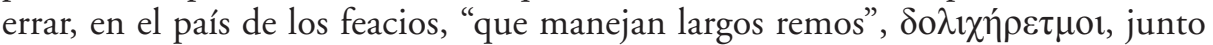
a los cuales iba a permanecer antes de emprender el regreso definitivo a su querida tierra de Ítaca.

\subsection{ODISEO EN ÍTACA. LA MNHETHPO-ФONIA (OD. 13-24).}

\subsubsection{Un paréntesis explicativo necesario}

Recordaremos, antes de acompañar a Odiseo y a la nave de los feacios a su llega-

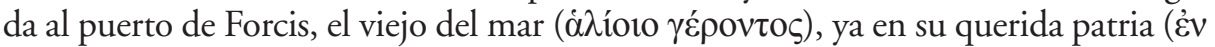

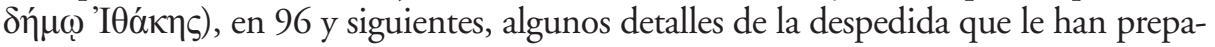
rado los feacios con su rey Alcínoo al frente. Es un comienzo in medias res, que no inicia un proemio ni tampoco un prólogo, como los que nos encontramos en las dos partes

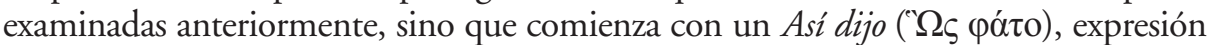
con la que se pretende recoger el final del relato de Odiseo en el canto anterior. En efecto, allí, en los versos finales, 447-453, Odiseo ya cansado, recuerda al rey Alcínoo y a su esposa Arete que ya anteriormente (canto 12, 254 ss.) les había relatado su llegada a Ogigia, junto a la ninfa Calipso, y su partida por mandato explícito de Zeus, con lo que les propone dar por concluido su relato y la transmisión poética igualmente fija el final del canto 12. Sin embargo, como podemos ver por el inicio del canto 13, en el que nos proponemos analizar la figura en estudio, este final, como a veces sucede, nos parece sorprendente, pues el canto 12 podría muy bien llegar, al menos ${ }^{20}$,

${ }^{20}$ Otra posibilidad en esta división entre el canto 12 y el 13 (que, por ejemplo, hemos visto, en cierto modo, propuesta en su traducción por Carles Riba: L'Odissea, 2014), nos podía llevar hasta el verso 184, tras el diálogo entre Zeus y su hermano Posidón sobre el castigo que se merecen los feacios. estos versos veríamos cómo Posidón, con el permiso de Zeus, convertirá la nave, que ha transportado 
hasta el verso 91, incluyendo, por tanto, estos versos en el 13, primero de la parte tercera, con los detalles de los preparativos, los regalos, el sacrificio de un buey a Zeus y las amables palabras de agradecimiento de Odiseo al rey Alcínoo, a su esposa Arete y a todos los feacios, que tan generosos han sido con él. Tras todo lo cual, Odiseo sube finalmente a la nave que le han preparado y, ya a bordo, se nos dice, vv. 79-80:

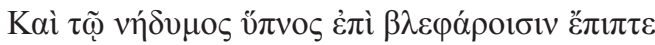

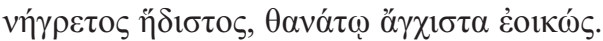
«Y sobre sus párpados le caía un profundo sueño, restaurador, dulcísimo, lo más parecido a la muerte».

A lo que sigue una bella comparación de corceles veloces con la nave, igualmente veloz, que lleva a un hombre, a Odiseo, v. 92:

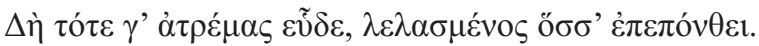
"que entonces, dormía ya tranquilo, olvidado de todo lo que había sufrido».

Un final, pensamos, más apropiado, para dar paso a dos bellísimos versos, muy del gusto homérico, con los que comienza el canto 13, y que analizamos más tarde, que dicen así, vv. 93-94:

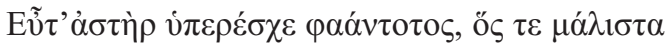

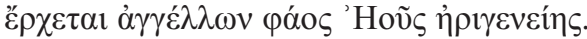

«Cuando se había alzado el astro que más brilla y que en lo más alto

avanza, anunciando la luz de Aurora matutina.»

\subsubsection{Análisis concreto de la que hemos titulado:}

Odiseo en Ítaca. La $\mu v \eta \sigma \tau \eta \rho-\varphi o v i ́ \alpha$.

Sin embargo, dejando a un lado lo anteriormente propuesto o, mejor, visto como división posible, nos vamos a situar en el principio del canto trece, adoptado por la tradición, ya que entre los versos 5 y 6 nos encontramos con un ejemplo de la figura en estudio, que nos parece contener algunos datos, en las palabras del rey Alcínoo, que, sin duda, merecen nuestra atención. Los citados versos dicen así:

a Odiseo a su tierra patria, en una roca, enraizándola en el fondo del mar. De este modo pondríamos como final del canto 12 el sacrificio de doce toros a Posidón por parte del rey Alcínoo y los feacios, para intentar que el dios no oculte la ciudad tras un monte. Así se situaría el comienzo del canto 13 en los versos 184-187, que servirían muy bien como punto de enlace entre los dos cantos, y, a la vez, nos trasladaría al estado en el que dejamos a Odiseo en el verso 92, durmiendo plácidamente en la nave feacia, en la que ahora despertaría, preguntándose en qué tierra de hombres se encontraba. 


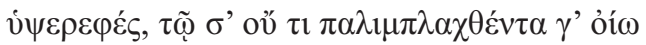

$\grave{\alpha} \psi \dot{\alpha} \pi 0 v 0 \sigma \tau \eta \dot{\sigma \varepsilon \imath v}$

«de elevado techo ${ }^{21}$, por eso pienso que tú, sin tener que andar más tiempo errante, de nuevo regresarás a casa,»

En efecto, tenemos aquí dos de los rasgos principales destacados por el poeta

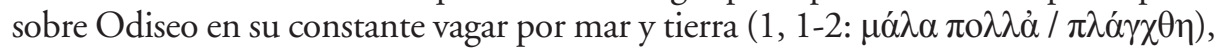

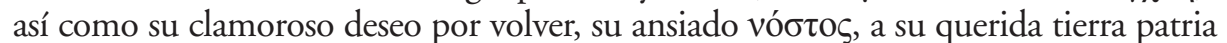

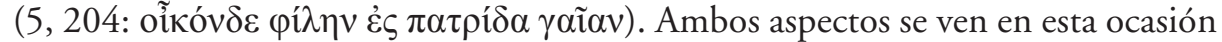
puestos de relieve con el empleo de la figura del encabalgamiento "integral necessary"

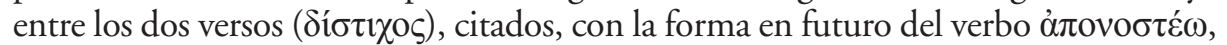
"regresar", prácticamente siempre "a casa" ${ }^{22}$, dependiendo, según traducimos nosotros, del verbo principal oî $\omega$ y el participio verbal, $\pi \alpha \lambda u \mu \pi \lambda \alpha \chi \theta \varepsilon \dot{\varepsilon} v \tau \alpha$, concertado con el sujeto, Odiseo, entendido por el hablante.

Tras este breve inciso, traslademos ahora nuestra atención a los versos en los que supuestamente colocaríamos ${ }^{23}$ el principio del canto 13 , ya que entre los versos analizados y estos versos, hemos recogido seis lugares de la figura en estudio, pero sin una relación clara y significativa ${ }^{24}$ (vv. 10-11; 14-15; 21-22; 26-27; $54-55$; 59-60), con el contenido de esta tercera parte de la Odisea. Los versos, ya anteriormente citados, son 93-94:

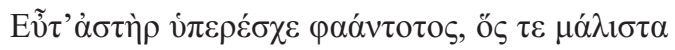

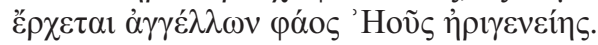

\footnotetext{
${ }^{21}$ Este epíteto se refiere al elevado techo del palacio de bronce, $\chi \alpha \lambda \kappa o \beta \alpha \tau \dot{\varepsilon} \zeta \delta \tilde{\omega}$, de Alcínoo, que hallamos en las orgullosas palabras del rey feacio en el verso anterior.

${ }^{22}$ Verbo pronunciado en su discurso por el rey Alcínoo, con el convencimiento de que sus hombres llevarán a Odiseo de regreso a su querida patria. Se trata, por lo demás, de una interpretación que aceptan la mayoría de los traductores nacionales y extranjeros frente a "regresar" de nuevo a Esqueria y al palacio de Alcínoo (cf. Autenrieth G. / A. Kaegi, A., 1999, Wörterbuch ..., a lo que podemos añadir los ejemplos en $I l .1,60$ y $O d$. 24, 471, e incluso en $I l .48,499$ y 12, 115, ya que se refieren a Ilio, por tanto a la casa de Héctor, e incluso en 17, 406, donde es Aquiles el que cree que su amigo Patroclo no volverá a su tienda, su casa en el campamento griego), como quiere algún traductor, interpretando la imposibilidad manifestada por el rey feacio de que Odiseo pueda volver a su palacio, dado su conocimiento del vaticinio, según el cual su ciudad se vería ocultada tras un gran peñasco, esto es, la nave, que había conducido a Odiseo, odiado por Posidón. La expresión "de elevado

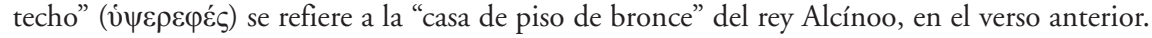

${ }^{23}$ Cf. la nota 14.

${ }^{24}$ Como tampoco la hemos hallado, como sucedió en las dos partes 1 y 2 de la Odisea, en los siguientes versos, en los que también se puede señalar un clara encabalgamiento, y que son los siguientes: 10-11; 14-15; 21-22; 26-27; 69-60; 107-108; 143-144; 205-206; 244-245; 272-273; 283-284; 285-286; 326-327 y 363-364. En una proporción de 14 a 22, donde constataremos en nuestro análisis posterior, que son más los lugares, con ocho de diferencia, donde la figura del encabalgamiento "integral necessary" en estudio señala y diríamos que resalta el contenido del canto, según la edición tradicional, prescindiendo de nuestro breve y necesario paréntesis sobre una posible variante para el comienzo del mismo.
} 
«Cuando se había alzado el astro que más brilla y que en lo más alto avanza, anunciando la luz de Aurora matutina»

con ellos el poeta, usando igualmente ${ }^{25}$ la figura del encabalgamiento "integral neces-

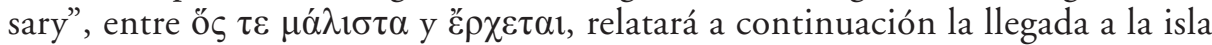
de la nave feacia y en ella a nuestro héroe, que finalmente podrá contemplar de nuevo

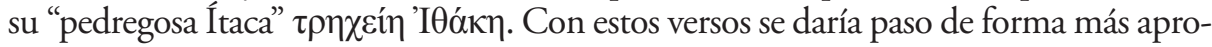
piada, diríamos, al canto 13 y con ellos al comienzo de la tercera parte del poema, que hemos titulado Odiseo en Ítaca. La $\mu v \eta \sigma \tau \eta \rho \varphi \varphi o v i a ~(O d .13-24)$. Aquí, en este

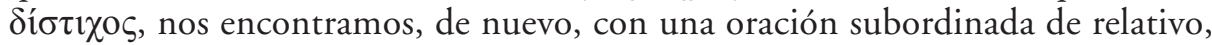
verso 93, completada en su sentido con la forma verbal ह̌p $\chi \varepsilon \tau \alpha$. Hacia ella, en efecto, y sin solución de continuidad, se ve arrastrada, en construcción paralela con la forma verbal anterior $\dot{\pi} \pi \varepsilon \rho \varepsilon \dot{\sigma} \sigma \varepsilon \varepsilon$, con la que comparte el mismo sujeto agente, un astro o estrella "que más brilla" ( $\varphi \alpha \alpha ́ v \tau o \tau o \varsigma)$. A su vez, el significado de Ě $\rho \chi \varepsilon \tau \alpha \iota$ se ve

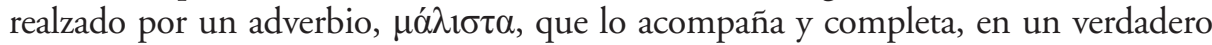
encabalgamiento "integral necessary", para anunciar la luz "de Aurora matutina", una de las personificaciones con las que el poeta adorna su relato y que como nombre propio, como hemos dicho ya anteriormente, traducimos sin artículo.

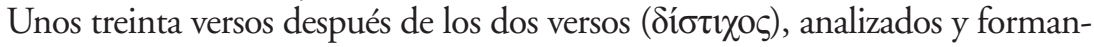
do un verdadero "nido" de contenido nos encontramos con cuatro lugares, como veremos, en sólo siete versos, cuyo contenido nos recuerda y hace presente la cólera y la decepción del dios Posidón ante el cariz que están tomando las noticias en torno a los avatares que rodean a su odiado enemigo, Odiseo, que finalmente ha llegado

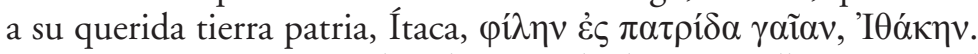

Veamos, pues, cuál es el contenido de este que llamamos "nido" de contenido:

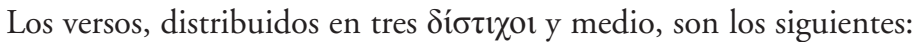

$125-126^{26} \operatorname{con} \lambda \eta \dot{\theta} \theta \varepsilon \tau$ ' $\dot{\alpha} \pi \varepsilon 1 \lambda \alpha \dot{\alpha} \omega v$, "se había olvidado de sus amenazas", el que sacude la tierra, Posidón y $126-127^{27} \operatorname{con} \pi \rho \tilde{\omega} \tau o v \varepsilon \dot{\pi} \eta \pi \varepsilon i ́ \lambda \eta \sigma \varepsilon$, "en un principio había amenazado."

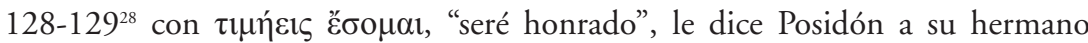
Zeus.131-13229 con oǐk $\alpha \delta$ ' غ̇ं $\varepsilon \varepsilon v ́ \sigma o \mu \alpha 1$, “iba a regresar a casa”. Odiseo está a punto

${ }^{25}$ Nótese, además, cómo el verso es dactílico en cuatro de sus seis pies, con un espondeo en el primero de los pies con la alusión al astro, que anuncia la luz de Aurora y con ello la llegada del nuevo día.

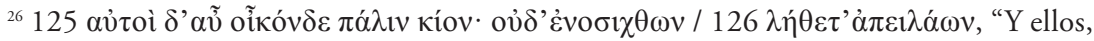
a su vez, marchaban de nuevo a casa. Pero el que sacude la tierra no se había olvidado de sus amenazas".

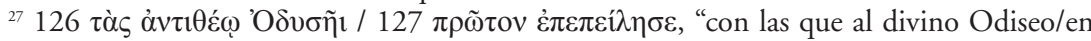
un principio había amenazado."

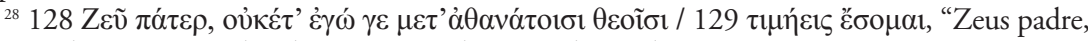
nunca más, incluso yo, entre los dioses inmortales / seré honrado"

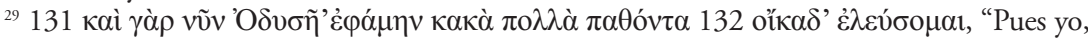
también ahora, había admitido que Odiseo, tras padecer muchos males, iba a regresar a casa". 
de regresar a casa con ayuda de los feacios, a pesar de la persecución de Posidón, que parece admitir su derrota ante su poderoso hermano, Zeus.

En los cuatro casos, el paso de un verso a otro, como podemos claramente constatar, se produce sin que haya signo alguno de puntuación entre ellos, con lo que está claro que nos encontramos con cuatro ejemplos ${ }^{30}$ de la figura del encabalgamiento "integral necessary", con la que se destaca, pensamos, junto a otras figuras de estilo, como la aliteración con presencia de $\pi$ (verso 127) y dominio de vocales (verso 128), numerosas marcas de elisión (en todos los versos citados) y alternancia de espondeos y dáctilos (igualmente en el verso 127) y con las constantes amenazas, la frustración final del dios ante el regreso, que no ha podido impedir, del odiado mortal, que había dejado ciego a su hijo Polifemo, así como la rivalidad entre los dioses olímpicos y la primacía entre todos ellos de Zeus, que el poeta parece presentarnos con un ligero matiz de imparcialidad ante los sucesos que suceden entre los mortales, y que, sin duda, en el caso concreto de Odiseo es importante, además de los insistentes ruegos a favor del itacense de su hija Palas, que se reve-

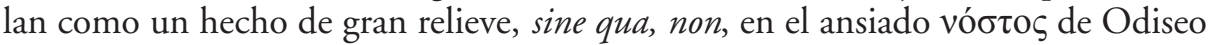
a su querida patria.

Sin embargo, no muy lejos, encontramos dos casos, versos $149-151^{31}$ y $175-177^{32}$, en los que se nos revela la venganza de Posidón contra el pueblo feacio,

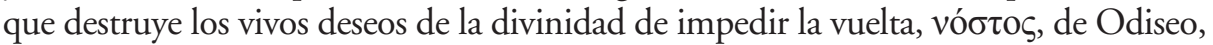
un verdadero Leitmotiv a lo largo de todo el poema. Esta cardinal circunstancia es recordada por el poeta de nuevo en esta ocasión, por lo que, dada su importancia en el relato del poema, pensamos que estaba justificada y, a la vez, era muy conveniente su cita en este lugar.

Siguiendo más adelante, nos encontramos con unos versos, en los que, primero, $187-188^{33}$, se nos muestra a Odiseo, rodeado de los regalos que le hicieron

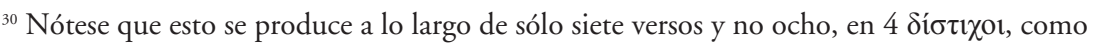
podía esperarse, con lo que el que hemos llamado "nido" de contenido, para expresar las amenazas de Posidón y su queja ante su hermano Zeus, quedan fuertemente destacadas.

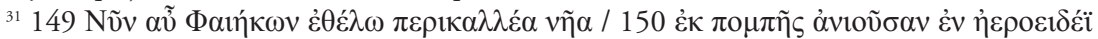

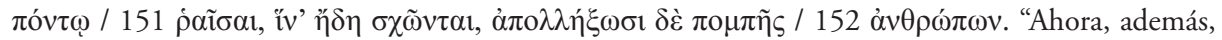
quiero destruir la hermosísima nave de los feacios / al volver del viaje de escolta en el brumoso ponto/ para que se contengan y terminen con la escolta / de hombres y quiero ponerles alrededor de la ciudad, ocultándola, una gran roca.

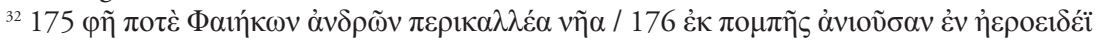

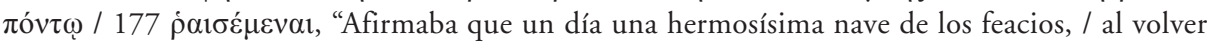
de una escolta en el umbroso mar/, destruiría".

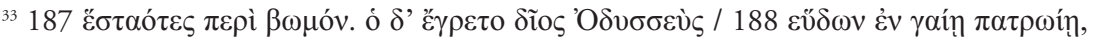

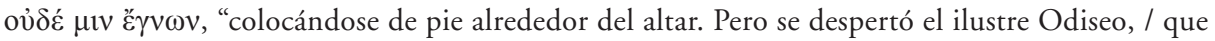
dormía ya en tierra patria, y no la reconoció.” 
los feacios ${ }^{34}$, al salir de Esqueria, despertándose en su tierra patria, tema nuclear de la tercer parte del poema; sólo unos pocos versos después, 203-204³5, la confusión de nuestro héroe, que no reconoce el lugar donde ha despertado; y a continuación, 211-212 $2^{36}$, las quejas que Odiseo profiere, al creerse engañado por los feacios, que, según le parece, no le han cumplido su promesa de llevarlo a su tierra. En los tres casos citados, además de un verso totalmente dactílico, 187, seguido de uno espondaico, excepto en el quinto pie, 188, o la alternancia entre espondeos y dáctilos en el 212, con lo que con esto puede querer resaltar nuestro poeta, hallamos igualmente la figura del encabalgamiento "integral necessary", con una forma verbal, que en los tres casos señala el extraño desconocimiento de Odiseo de su propia tierra y la esplendidez de sus huéspedes feacios, preparándonos para los importantes acontecimientos de esta parte de la Odisea, donde el aedo nos va disponiendo para los principales acontecimientos, que van a suceder en ella, como son: el encuentro con su porquerizo Eumeo, con su hijo Telémaco, con su mujer y, sobre todo, con su venganza sobre los soberbios y altaneros pretendientes de Penélope.

Con Odiseo ya auxiliado, una vez más, por su diosa protectora, Atenea, él puede ya reconocer la gruta, $\sigma \pi \varepsilon ́ o \varsigma$, amplia y abovedada, donde solía rendir culto a las ninfas, $349-350^{37}$, y a las que, cuando ya Atenea le haya quitado la sombra de los ojos y él haya reconocido finalmente su tierra patria, saludará con plegarias unos versos más adelante, 357-358 38 ; en ambos casos el uso de la figura en estudio es diferente, pero su misma diferencia resalta el sentido especial del pasaje, con un

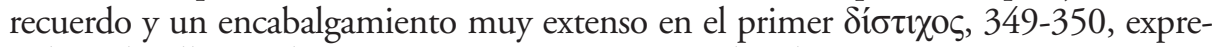
sado todo ello en el primer pie, 349 , con cinco dáctilos, mientras que son cuatro los espondeos en el segundo, 350, y una sola forma verbal, $\chi \alpha i^{\prime} \rho \varepsilon \tau^{\prime}(\mathrm{o})$ en el segundo

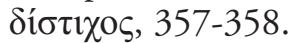

Finalmente en los versos $409-410^{39}$, Odiseo va a encontrar a su fiel criado, el porquero Eumeo, tan importante en esta parte final del poema, canto 21, en el

${ }^{34} \mathrm{~A}$ los que se referirá en los versos 217-218, como bellísimos trípodes y calderas, y a los que volverá a aludir en los versos 283-284, en su relato al joven (Atenea), que le sale al paso y a los que Atenea vuelve a nombrar en 304-305 y en 363-364 le recomienda a su protegido colocar en la gruta de las ninfas.

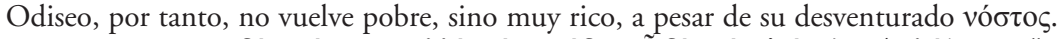

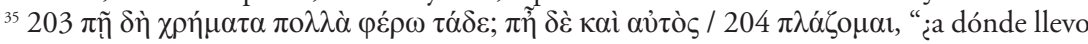
ahora estas innumerables riquezas? ¿Y yo mismo hacia dónde voy a ir errante?

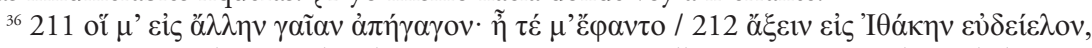

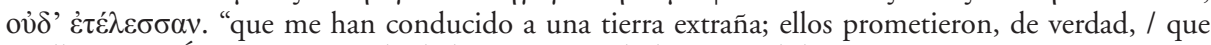
me llevarían a Ítaca, que se ve desde lejos, pero no lo han cumplido."

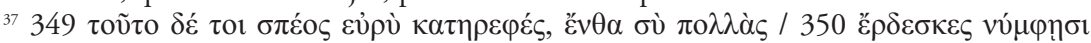

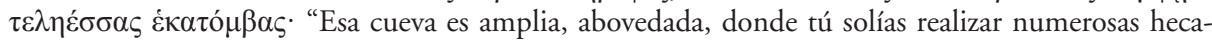
tombes perfectas a las ninfas".

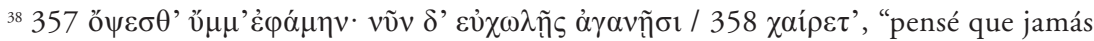
os volvería a ver, mas, ahora, con amables plegarias / os saludo".

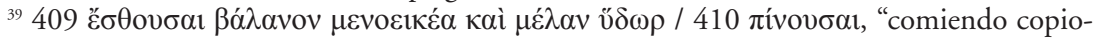
sa bellota y obscura agua / bebiendo". 
reconocimiento de Odiseo, y en el 22 en la matanza de los pretendientes y siervos traidores. En esta ocasión el fiel criado cumple con su trabajo, dando de beber obscura agua a los cerdos de su señor, a los que él cuida, pensando siempre en su regreso. Ya al final de este canto trece Atenea le dice a Odiseo que ella, mientras él visita al porquerizo Eumeo, irá a Esparta para ordenar a su hijo Telémaco que vuelva a su casa y que ella lo librará de la trampa que le preparan los pretendientes. El hijo de Odiseo recordamos que se fue en la primera parte del poema a las cortes de Néstor y Menelao a Pilo y Lacedemonia, respectivamente, a buscar noticias sobre su padre, a cuya última corte, la ilustre Lacedemonia, $\dot{\varepsilon} \varsigma \Lambda \alpha \kappa \varepsilon \delta \alpha \mu o v \alpha \delta \tilde{\alpha} \alpha v$, le comunica la diosa a Odiseo en los versos $414-415^{40}$, que ella irá para ordenarle que regrese. El canto finaliza, transformando Atenea a su protegido en un mendigo, cubriéndolo con una túnica y manto andrajosos, para que no sea reconocido por los pretendientes ni por su esposa Penélope y marchándose la diosa, versos 439-440, a la corte de Menelao, anteriormente mencionada.

\section{CONCLUSIONES}

Llegamos, por último, al final de nuestro arduo trabajo, después de enfrentarnos al análisis de aquellos versos elegidos, en los que el uso del encabalgamien-

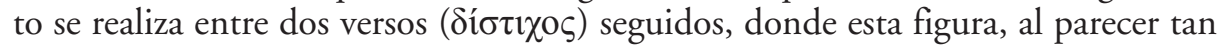
amada por el poeta, el desbordamiento hacia el verso siguiente, está representada por una forma verbal ${ }^{41}$, que, sobre todo o, quizá mejor, significativamente, se relaciona con el contenido principal de cada una de las partes, en las que hemos aceptado la división de la Odisea. Además, allí donde la acompañan, hemos señalado otras formas de estilo muy propias de la épica homérica, como la aliteración, el hipérbaton, la Ringkomposition, la métrica, etc., que, sin duda, nos sitúan ante un texto compuesto entre los ejemplos más significativos de la llamada "oral poetry", y que en el seguidor de Homero e imitador romano más excelso, Virgilio ${ }^{42}$, encontraremos con una frecuencia muy significativa ${ }^{43}$. Así, en una proporción de 7 a 6 , en el canto

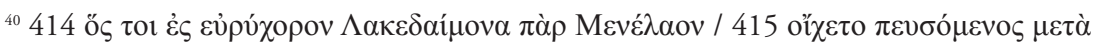
$\sigma o ̀ v ~ \kappa \lambda \varepsilon ́$ os "que se ha ido a la espaciosa Lacedemón, a casa de Menelao/ para buscar noticias tuyas".

${ }^{41}$ Por razones de espacio, ya hemos señalado que dejamos fuera de nuestro análisis aquellos casos en los que la figura en estudio se realiza por medio de sustantivos, adjetivos, adverbios y preposiciones, que sí hemos recogido. Sólo añadir que en los casos en los que el encabalgamiento se realiza por medio de un nombre común o propio la proporción en los tres cantos es muy superior en aquellos lugares en los que se destaca un sujeto relacionado con el tema tratado, en una proporción de 8 a 5 , en el canto primero, de 16 a 5, en el canto cinco y, finalmente, de 12 a 6, en el canto 13 .

${ }_{42}$ No en vano es elegido acertadamente por G. S. Kirk, op. cit., pp. 85-112, para comparar y ejemplificar el tema de su obra, Homero y la tradición oral, junto al gran poeta inglés John Milton y su The Paradise Lost.

${ }^{43}$ Así, véase Meister, K., op. cit. p. 28, nota 1, donde podemos leer: Man vergleiche um des Kontrastes willen den homerischen Versbau mit dem der Äneis, wo das Enjambament haüfig ist, es decir, 
primero, con mayoría escasa de casos, llamemos positivos; de 8 a 13, en el canto quinto, con clara superioridad numérica de los casos en los que no hemos podido señalar un contenido relativo al tema en él tratado y, finalmente, de 22 a 14, con mayoría de aquellos casos en los que el poeta para resaltar el tema, que en cada canto ocupa su atención, recurre, como hemos intentado demostrar a lo largo de nuestro numérico y explicativo trabajo, al llamado por Kirk "Enjambment integral necessary", se produce cuando el significado del verso anterior, sin coma, se desborda al verso siguiente, empleando una forma verbal. Es decir, que será siempre esclarecedor y positivo, en cualquier estudio del estilo en las obras épicas, no sólo escritas en griego, a la hora de analizar su estructura poética, tener en cuenta este poético instrumento, que en la Odisea el gran poeta Homero nos ha mostrado como un instrumento de gran valor, no sólo para resaltar el significado por él elegido, sino también, en el caso que nos ocupa, la unidad entre las tres partes estudiadas.

\section{REFERENCIAS BIBLIOGRÁFICAS}

Autenrieth, G. / KaEgI, A. (1999): Wörterbuch zu den Homerischen Gedichten, 14. Auflage, Neudruck der von Adolf KAEGI besorgten 13 vielfach verbesserten Auflage (1920), mit einem Geleitwort von Joachim LATACZ und einer Einleitung von Andreas WILI, B. G. Teubner, Stuttgart und Leipzig.

BAKKER, Egbert J. (1990): «Homeric Discourse and Enjambement: A Cognitive Approach», TAPhA 120: 1-21.

BARnes, Harry R. (1979): «Enjambement and Oral Composition», TAPhA 109: 1-10.

BASSETT, S. E. (1926): «The So-Called Emphatic Position of the Runover Word in the Homeric Hexameter», TAPhA 57: 116-148.

Bassett, S. E. (1938 [2003 $\left.\left.{ }^{12}\right]\right)$ : The Poetry of Homer, U. of California Press, Berkeley.

Clark, Matthew (1984): «Enjambment and Binding in Homeric Hexameter», Phoenix 48 (2 [Summer]): 95-114.

Clayman, Dee Lesser \& VAN NORWICK, Thomas (1977): «Enjambement in Greek Hexameter Poetry», TAPhA 107: 85-92 [que critican la obra de M. Parry].

en una comparación del verso en la poesía homérica con el hexámetro de la Eneida, en este poema virgiliano es más frecuente el uso de la figura del Enjambement. Además no es de extrañar que sean versos de este gran poeta romano los que con gran frecuencia sean elegidos para ejemplificar algunas de estas figuras recogidas por nosotros en la Odisea, entre las que se nos permitirá recordar la aliteración en aquél delicado verso de Egl. 5. 38, que decía: pro molli niola pro purpureo narcisso, --, -vu, $--,-v v,--,--$, con dominio de vocales de timbre suave, abierto o cerrado y con alternancia de pies dáctilos y espondeos, siendo el quinto también espondeo, con lo que esto puede significar, o el también célebre primer verso de su Eneida: Arma uirumque cano, Troiae qui primus ab oris, -vu, -vu, --, --, -vu, -- que empieza con ritmo rápido, en dáctilos, para detenerse en cano, Troiae, qui, con dos espondeos, -nō Troì lae ūi, que resaltan la acción del poeta, su cantar, cano, y el nombre de la ciudad de donde viene, Troiae. Pero, igualmente encontramos el encabalgamiento en numerosos lugares, como acabamos de señalar en nota anterior, y entre los que sólo señalaremos como posibles ejemplos también del canto primero de la Eneida la pareja de versos 10-11 con impulerit y 31-32 con errabat. 
DukAT, Zdeslav (1991): «Enjambement as a Criterion for Orality in Homeric and South Slavic epic Poetry», Oral Tradition 6 (2-3): 303-315 [publicada como Journal at Harvard University, desde 1986].

Halicarnaso, Dionisio de (1978): De compositione uerborum, París.

Higbie, C. (1990): Messure and Music: Enjambement and Sentence Structure in the Iliad, Clarendon Press, Oxford.

KIRK, G. S. (1976): Homer and the Oral Poetry, University Press, Cambridge.

LONGINO Rhetor (1968): De Sublimitate, Oxford.

MeISTER, Karl (1921): Die homerische Kunstsprache [Classic Reprint, Forgotten Books, London 2018] Leipzig.

PARRY, Milman (1929): «The distinctive Character of Enjambement in Homeric Verse», TAPhA 60: 200-220.

PARRY, Adam (ed.) (1987): The Making of Homeric Verse, Oxford University Press, Oxford 1, pp. 251-265 [donde, entre otros trabajos de su padre Milman, se recoge el artículo sobre el Enjambement, antes citado].

SEEK, G. A. (2004): Homer. Eine Einfuihrung, Reclam, Stuttgart. 